\title{
Incidencia y Tipo de Istmos en Primeros Molares Permanentes Humanos, Evaluación in vitro
}

\author{
Incidence and Type of Isthmus in Human Permanent First Molars, in vitro Assessment \\ Ricardo Oliva Rodríguez; Anna Gabriela Gastélum Zazueta²; Yolanda Hernández Molinar; \\ Jairo Mariel Cárdenas ${ }^{1}$; Francisco Javier Gutiérrez Cantú ${ }^{1} \&$ Daniel Silva-Herzog Flores ${ }^{1}$
}

OLIVA, R. R.; GASTÉLUM, Z. A. G. ; HERNÁNDEZ, M. Y.; MARIEL, C. J.; GUTIÉRREZ, C. F. J. \& SILVA-HERZOG, F. D. Incidencia y tipo de istmos en primeros molares permanentes humanos, evaluación in vitro. Int. J. Morphol., 35(4):1280-1284, 2017.

RESUMEN: El conocimiento de la complejidad anatómica del sistema radicular de conductos generada por conductos accesorios, deltas apicales e istmos, aumenta la probabilidad de éxito en el tratamiento endodóntico, dado que dichos sitios pueden ser un reservorio de microorganismos y materia orgánica que pueden limitar la acción de los instrumentos, las sustancias irrigantes y los medicamentos intraconducto. El objetivo de este estudio fue determinar la incidencia y el tipo de istmos presentes en los distintos segmentos de raíces mesio-vestibulares de molares superiores y mesiales de molares inferiores. Se recolectaron setenta y dos primeros molares extraídos, treinta y seis superiores y treinta y seis inferiores a los cuales se les tomó radiografías y se les estudiaron distintas variantes anatómicas como número de conductos, curvatura y configuración radicular; posteriormente se les eliminó la corona y las raíces mesio-vestibulares de superiores y mesiales de inferiores se incluyeron en resina epóxica y con un disco de diamante se hicieron cortes transversales en cada raíz dejando cinco secciones de un mm iniciando desde el extremo apical hasta el cervical, se tiñeron con cristal violeta y se analizó la presencia de istmos y su clasificación bajo microscopio estereoscópico. Los resultados mostraron una alta incidencia de istmos asociada a raices de configuración aplanada. Entre más cerca del ápice, más baja la incidencia de istmo tanto en las raices mesiovestibulares del primer molar superior como en las raices mesiales del primer molar inferior; la más alta incidencia de istmos se dio aproximadamente a siete mm del ápice y la clase de istmo más frecuentemente encontrada fue la clase II en ambos tipos de raíz.

PALABRAS CLAVE: Istmo; Anatomía; Molar; Endodoncia.

\section{INTRODUCCIÓN}

El éxito en la terapia endodóntica se basa en gran medida en el conocimiento de la morfología y las complejidades anatómicas de los conductos radiculares, tales como conductos accesorios, deltas apicales y muy especialmente la presencia de istmos. Se define como istmo a un espacio en forma de banda estrecha que comunica completa o parcialmente a dos conductos radiculares dentro de una misma raíz con contenido de tejido pulpar (Weller et al., 1995).

El Istmo es una característica anatómica importante debido a que puede contener restos de pulpa, tejidos necróticos así como microorganismos y sus subproductos que pueden promover la reinfección luego del tratamiento de conductos.

Una gran cantidad de estudios se han llevado a cabo para determinar la prevalencia de istmos en molares hu- manos bajo distintas metodologías; algunas de ellas seccionando longitudinal o transversalmente las raíces para observarlas al microscopio, ya sea estereoscópico, de disecciones, electrónico de barrido o incluso microscopio quirúrgico (Kontakiotis et al., 2010; Bao et al., 2012); tiñéndolas y embebiéndolas en resinas para posteriormente seccionarlas o diafanizando las raíces para evidenciar el istmo sin seccionar la raíz, y más recientemente se ha hecho uso de tecnologías como la endoscopía o la tomografía tanto de haz cónico como micro computarizada para tal fin (von Arx, 2005; Pécora et al., 2013; Estrela et al., 2015; Versiani et al., 2016). Otro punto de variación en los distintos estudios es la cantidad de segmentos analizados de cada raíz, muchos trabajos han estudiado la presencia de istmo en los tercios apical, medio y cervical mientras que otros la analizan en hasta 5 o 6 secciones de $1 \mathrm{~mm}$ de espesor para cada raíz.

\footnotetext{
${ }^{1}$ Maestría en Endodoncia, Facultad de Estomatología, Universidad Autónoma de San Luis Potosí, San Luis Potosí, México. ${ }^{2}$ Facultad de Odontología, Universidad Autónoma de Sinaloa, Sinaloa, México.
} 
Se sabe que existe una alta incidencia de istmo en las raíces de configuración aplanada que tienden a presentar dos o más conductos radiculares, especialmente en las raíces mesio-vestibulares de molares superiores y sobre todo en la raíz mesial de los primeros molares inferiores. El istmo de acuerdo a sus características anatómicas se clasifica en cinco categorías de acuerdo a Hsu \& Kim (1997): Tipo I: Son dos conductos separados donde no aparece la unión de ambos conductos; en el Tipo II se observa la unión entre los dos conductos separados conectados por el istmo; en el Tipo III se encuentran tres conductos conectados por un istmo, mientras que en el Tipo IV hay dos conductos alargados y unidos en el centro; y finalmente en el Tipo $\mathrm{V}$ hay un conducto único muy amplio y alargado (Fig. 1).

El principal objetivo de este estudio fue evaluar la incidencia, el tipo y posición de istmos presentes en primeros molares superiores e inferiores humanos extraídos.

\section{MATERIAL Y MÉTODO}

Se recolectaron 72 molares en total, 36 primeros molares superiores y 36 primeros molares inferiores, sin evidencia de reabsorción radicular, tratamiento de conductos, fracturas o ápices abiertos y se almacenaron en formalina al 10 $\%$. Después de lavarlos con agua y secarlos se identificó cada pieza con un código específico y se les tomaron radiografías periapicales en sentido vestíbulo-palatino o vestíbulo-lingual y mesio-distal con un tiempo de exposición de $0.5 \mathrm{~s}$. dirigiendo el cono en forma perpendicular a la película. Para obtener y registrar datos y características de su anatomía interna radicular así como su longitud total, longitud radicular, fusión de raíces, número de conductos, configuración radicular, ya sea cónica o aplanada y curvatura radicular.

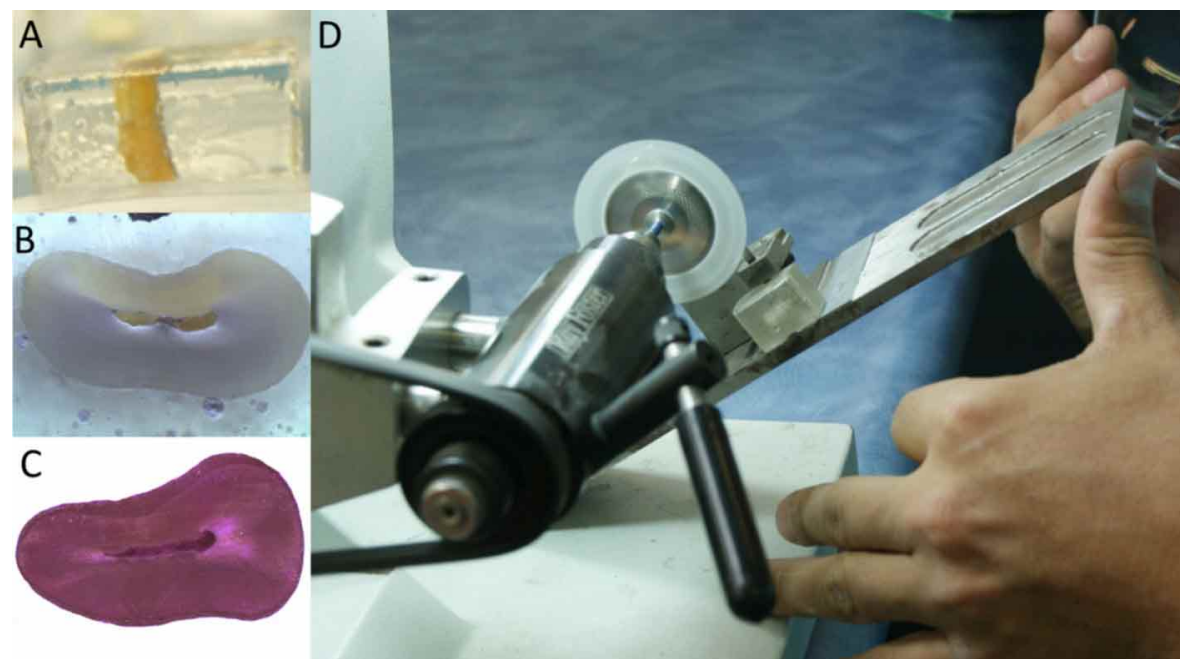

Los 72 molares se cortaron a nivel de la unión esmalte-cemento con un disco de carburo de baja velocidad, desechando la corona, después se separó la raíz mesial de los molares inferiores y la mesio-vestibular de los superiores y a cada raíz se le otorgó un código identificador. Se colocó cada raíz en moldes de silicón, para embeberlas en resina epóxica (Fig. 1A), y posteriormente con un disco de diamante de baja velocidad (Fig. 1D) se hicieron cortes transversales (Fig. 1B), perpendiculares al eje axial de la raíz, haciendo segmentos aproximadamente de $1 \mathrm{~mm}$, iniciando desde el extremo apical hasta el cervical, correspondiendo el número 1 al segmento apical y el 5 al segmento cervical (Fig. 2). Las superficies seccionadas se tiñeron con cristal violeta durante 60 segundos (Fig. 1C), posteriormente se lavaron con agua corriente, se secaron y finalmente se observaron en el microscopio estereoscópico a 20x, cada imagen se almacenó digitalmente y un observador independiente evaluó las imágenes para determinar la presencia o ausencia de istmo, así como su clasificación morfológica basada en los criterios de Hsu \& Kim.

Fig.1. A. Raíz embebida en bloque de resina epóxica. B. Segmento de raiz resultante del corte con disco de diamante. C. Segmento de raiz teñida con cristal violeta. D. Ilustración del dispositivo utilizado para el corte de los bloques y obtención de los segmentos a evaluar al microscopio estereoscópico. 


\section{RESULTADOS}

La Tabla I muestra los datos resultantes de la observación macroscópica de los primeros molares superiores, dentro de los que resalta una muy baja incidencia de fusión radicular con un 5,55\%; un $50 \%$ de órganos dentarios con cuatro conductos, con presencia del cuarto conducto en la raíz mesiovestibular así como una marcada tendencia a mostrar curvatura radicular hacia distal hasta en un 83,3\% de los casos.

De la observación del primer molar inferior se puede destacar cómo a pesar de la configuración aplanada de la raíz distal hasta en un $86,11 \%$ de las ocasiones, la presencia de un conducto adicional en dicha raíz solo llega hasta un $11 \%$ de las muestras analizadas; por otro lado se observa una ausencia total de fusión radicular y una curvatura hacia distal en un 93,36 \% de los casos (Tabla II).

En la Tabla III se muestran los resultados concernientes a las 36 raíces mesiovestibulares de los molares superiores según la sección del diente estudiada en términos de presencia o ausencia de istmo y a qué clase pertenecen los istmos encontrados en los distintos segmentos radiculares.

La incidencia de istmo en la raíz mesiovestibular aumentó consistentemente desde un $50 \%$ para el segmento apical 1 hasta un 88,88\% para el segmento cervical 5. Mientras que la clase de istmo más prevalente para esta raíz fue la clase II en los 5 distintos segmentos radiculares y la más escasa fue la clase III.

Se observó en las raíces mesiales de los molares inferiores una alta incidencia de istmo iniciando con un $63 \%$ en el segmento 1 , aumentando rápidamente su presencia a partir del segmento 2 hasta llegar a un $100 \%$ tanto en el segmento 4 como en el 5; mientras que en términos de cuál de los tipos de istmo predomina en esta raíz se pudo observar nuevamente que la clase más frecuentemente encontrada fue la II y la menos frecuente fue la clase III, tal y como ocurrió en la raíz mesiovestibular del molar superior (Tabla IV).

Tabla I. Datos resultantes de la observación macroscópica del primer molar superior.

\begin{tabular}{lll}
\hline & Primer Molar Superior $(\mathrm{n}=36)$ & \\
\hline Longitud total & Promedio $(19,7 \mathrm{~mm})$ & Rango $(17-24 \mathrm{~mm})$ \\
Longitud radicular & Promedio $(14,1 \mathrm{~mm})$ & Rango $(12-16 \mathrm{~mm})$ \\
Fusión radicular & Ausente $94,45 \%$ & Presente 5,55\% \\
Forma radicular & Cónica & Aplanada \\
- Palatina & $100 \%$ & $0 \%$ \\
- Mesiovestibular & $0 \%$ & $100 \%$ \\
- Distovestibular & $69,94 \%$ & $30,06 \%$ \\
Dirección de curvatura & Hacia Distal & $83,33 \%$ \\
& Hacia Mesial & $8,33 \%$ \\
& Sin curvatura & $8,33 \%$ \\
$\mathrm{~N}^{\circ}$ de conductos & 3 Conductos $(50 \%)$ & 4 Conductos $(50 \%)$ \\
\hline
\end{tabular}

Tabla II. Datos resultantes de la observación macroscópica del primer molar inferior.

\begin{tabular}{|c|c|c|}
\hline \multicolumn{3}{|c|}{ Primer Molar Inferior $(n=36)$} \\
\hline Longitud total & Promedio $(20 \mathrm{~mm})$ & Rango (16-23 mm) \\
\hline Longitud radicular & Promedio $(15,5 \mathrm{~mm})$ & Rango (10-19 mm) \\
\hline Fusión radicular & Ausente $100 \%$ & Presente $0 \%$ \\
\hline Configuración radicular & Cónica & Aplanada \\
\hline - Raíz Mesial & $0 \%$ & $100 \%$ \\
\hline - Raíz Distal & $13,88 \%$ & $86,11 \%$ \\
\hline Dirección de curvatura & Hacia Distal & $93,36 \%$ \\
\hline & Hacia Mesial & $6,64 \%$ \\
\hline $\mathrm{N}^{\circ}$ de conductos & 2 Conductos $(5,55 \%)$ & 4 Conductos $(11,11 \%)$ \\
\hline
\end{tabular}


Tabla III. Posición del istmo a lo largo de los segmentos radiculares y distribución de acuerdo a su clasificación morfológica en la raíz mesiovestibular del primer molar superior.

\begin{tabular}{cccccccc}
\hline \multicolumn{7}{c}{ Raíz Mesiovestibular de Primer Molar Superior (n=36) } \\
\hline Segmento & Sin Istmo (\%) & Con Istmo (\%) & \multicolumn{5}{c}{ Clase (\%) } \\
\cline { 4 - 7 } & & & I & II & III & IV & V \\
\hline 1 & $18(50 \%)$ & $18(50 \%)$ & 16,66 & 44,44 & 0 & 16,66 & 22,22 \\
2 & $8(22,22 \%)$ & $28(77,77 \%)$ & 0 & 46,42 & 0 & 14,28 & 39,28 \\
3 & $6(16,66 \%)$ & $30(83,33 \%)$ & 10 & 30 & 0 & 20 & 40 \\
4 & $5(13,88 \%)$ & $31(86,11 \%)$ & 0 & 32,25 & 6,45 & 29,03 & 32,25 \\
5 & $4(11,11 \%)$ & $32(88,88 \%)$ & 3,12 & 37,5 & 3,12 & 31,25 & 25 \\
\hline
\end{tabular}

Tabla IV. Posición del istmo a lo largo de los segmentos radiculares y distribución de acuerdo a su clasificación morfológica en la raíz mesial del primer molar inferior.

\begin{tabular}{cccccccc}
\hline \multicolumn{7}{c}{ Raíz Mesial de Primer Molar Inferior (n=36) } \\
\hline Segmento & S in Istmo (\%) & Con Istmo (\%) & \multicolumn{5}{c}{ Clase (\%) } \\
\cline { 4 - 7 } & & & I & II & III & IV & V \\
\hline 1 & $13(36.11 \%)$ & $23(63.88 \%)$ & 8.69 & 39.13 & 8.69 & 4.34 & 39.13 \\
2 & $3(8.33 \%)$ & $33(91.66 \%)$ & 9.09 & 33.33 & 6.06 & 21.21 & 30.80 \\
3 & $2(5.55 \%)$ & $34(94.45 \%)$ & 11.76 & 38.23 & 5.88 & 23.52 & 20.58 \\
4 & $0(0 \%)$ & $36(100 \%)$ & 8.33 & 41.66 & 2.77 & 38.88 & 8.33 \\
5 & $0(0 \%)$ & $36(100 \%)$ & 19.44 & 36.11 & 2.77 & 38.88 & 2.77 \\
\hline
\end{tabular}

\section{DISCUSIÓN}

Ha habido una gran cantidad de estudios de incidencia y tipo de istmo en distintas piezas dentales y raices de las mismas, todos basados en distintas metodologías. La metodología utilizada en el presente trabajo se basa en buena medida en la propuesta por Teixeira et al. (2003). El conocimiento profundo de la configuración interna del sistema de conductos radiculares es una de las herramientas que podrán conducir a la implementación de las mejores prácticas asociadas a la limpieza y conformación del espacio pulpar y también a un abordaje más eficiente cuando se planifica una cirugía apical.

En este trabajo fue posible evidenciar una alta incidencia de istmo en ambos tipos de raíz, la mesiovestibular del primer molar superior mostrando hasta un $88 \%$ de presencia de istmo y la mesial del primer molar inferior con hasta un $100 \%$ lo cual coincide con los hallazgos de Cleghorn et al. (2006) y de Pablo et al. (2010) quienes reportaron la incidencia en la raíz MV desde 76 hasta $100 \%$ y aproximadamente un $83 \%$ en la raíz mesial del primer molar inferior.

La presencia del cuarto conducto en el primer molar superior ascendió a $50 \%$ en el presente trabajo, lo cual parece ajustarse al estándar encontrado en la mayor parte de la literatura dado que Hess (1925) reportó la presencia de cuatro conductos en $54 \%$ de molares superiores, mientras que Falcão et al. (2016) mostró que el conducto mesiopalatino estaba presente en 56,25\% de los casos, y Pineda \& Kuttler (1972) re- portaron una incidencia de $51,5 \%$ de conductos adicionales en el primer molar superior lo cual se convierte en un dato muy relevante desde el punto de vista clínico.

La forma aplanada en el $100 \%$ de las raíces mesiovestibulares facilitaría la presencia de dos conductos dentro de esa raíz y al mismo tiempo la presencia de un istmo relacionando a ambos conductos, enfatizando la decisión de estudiar específicamente esta raíz en función de la presencia de istmo, mientras que haber alcanzado un $30 \%$ de configuración aplanada en la raíz distovestibular en el presente estudio, no implicó la presencia de un conducto adicional en ella, dado que la presencia de dos conductos en la raíz distovestibular es extremadamente escasa; Cleghorn et al. mediante una revisión de 14 estudios que incluyeron 2576 dientes informó que la configuración más común del sistema de conductos de la raíz distovestibular fue la de un solo conducto en $98,3 \%$, de dos conductos en $1,7 \%$ y de un solo foramen apical en $98 \%$.

En este trabajo, se encontró una alta cantidad de istmos en la raíz mesiovestibular del primer molar superior a una distancia de 3-7 mm desde el ápice, lo cual concuerda con Lima et al. (2014) y Tam \& Yu (2002). Del mismo modo la raíz mesial del molar inferior mostró una prevalencia muy alta de istmo desde los 3 hasta los $7 \mathrm{~mm}$ desde el ápice llegando hasta un $100 \%$ en el segmento 4 coincidiendo con los hallazgos de Gu et al. (2009). 
El istmo clase II fue el más frecuentemente encontrado en los dos tipos de raíz estudiadas en este trabajo; dicho tipo de istmo no muestra una comunicación franca entre los dos conductos, lo cual sugiere una obliteración de ese espacio por aposición dentinaria, aunque esto no debiera ocasionar el no poner énfasis en la limpieza a conciencia de esa área y la gran dependencia que se tiene de la irrigación con volúmenes copiosos de irrigante, sustancias irrigantes con adecuadas capacidades antimicrobianas y de disolución de materia orgánica y la potenciación del proceso mediante el uso de ultrasonido o presión apical negativa para conseguir un óptimo resultado de limpieza de la totalidad del espacio del sistema radicular de conductos, lo cual pueda conducir a elevar la expectativa de un pronóstico favorable de los tratamientos endodóntico y quirúrgico en el mediano y largo plazo.

Se concluye que se encontró una alta incidencia de istmos asociada a raices aplanadas. Entre más cerca del ápice, más baja la incidencia de istmo tanto en las raices mesiovestibulares del primer molar superior como en las raices mesiales del primer molar inferior, la más alta incidencia se notó a aproximadamente $7 \mathrm{~mm}$ del ápice y la clase de istmo más frecuentemente encontrada fue la clase II en ambos tipos de raíz.

OLIVA, R. R.; GASTÉLUM, Z. A. G. ; HERNÁNDEZ, M. Y.; MARIEL, C. J.; GUTIÉRREZ, C. F. J. \& SILVA-HERZOG, F. D. Incidence and type of isthmus in human permanent first molars, in vitro assessment. Int. J. Morphol., 35(4) :1280-1284, 2017.

SUMMARY: The profound knowledge of the root canal anatomical complexity generated by accessory canals, apical deltas and isthmuses, increases the likelihood of success in endodontic treatment, given that such sites may be a reservoir of microorganisms and organic matter that may limit the action of instruments, irrigant substances and intracanal medications. The objective of this study was to determine the incidence and type of isthmus in the various segments of mesio-buccal roots of first upper molars and mesial root of first lower molars. Seventy-two extracted first molars, thirty-six upper and thirty-six lower were radiographed and different anatomic variants such as number of canals, curvature and root configuration were studied. Later, mesio-buccal and mesial roots were cut and embedded in resin and sectioned with a diamond disc, five serial horizontal sections were made perpendicular to the root long axis. These were stained with crystal violet and examined under stereomicroscope in order to determine the presence of isthmuses and their classification. Results showed a high incidence of isthmus associated with roots of flattened configuration. The closer to the apex, the lower the incidence of isthmus observed in both in the upper and lower roots; the isthmus were most frequently found at $7 \mathrm{~mm}$ from the apex, the type of isthmus found more frequently was type II in both types of root.

KEY WORDS: Isthmus; Anatomy; Molar; Endodontics.

\section{REFERENCIAS BIBLIOGRÁFICAS}

Bao, X. D.; Yao, N.; Guo, H. J.; Zhang, C. F. \& Yue, L. Disinfection effects of MTAD on apical isthmus of the root canal system: a scanning electron microscopic evaluation. Beijing Da Xue Xue Bao, 44(1):34-8, 2012.

Cleghorn, B. M.; Christie, W. H. \& Dong, C. C. Root and root canal morphology of the human permanent maxillary first molar: a literature review. J. Endod., 32(9):813-21, 2006

de Pablo, O. V.; Estevez, R.; Péix Sanchez, M.; Heilborn, C. \& Cohenca, N. Root anatomy and canal configuration of the permanent mandibular first molar: a systematic review. J. Endod., 36(12):1919-31, 2010.

Estrela, C.; Rabelo, L. E.; de Souza, J. B.; Alencar, A. H.; Estrela, C. R.; Sousa Neto, M. D. \& Pécora, J. D. Frequency of root canal isthmi in human permanent teeth determined by Cone-beam computed tomography. J. Endod., 41(9):15359, 2015.

Falcão, C. A.; Albuquerque, V. C.; Amorim, N. L.; Freitas, S. A.; Santos, T. C.; Matos, F. T. \& Ferraz, M. A. Frequency of the mesiopalatal canal in upper first permanent molars viewed through computed tomography. Acta Odontol. Latinoam., 29(1):54-9, 2016.

Gu, L.; Wei, X.; Ling, J. \& Huang, X. A microcomputed tomographic study of canal isthmuses in the mesial root of mandibular first molars in a Chinese population. J. Endod., 35(3):353-6, 2009.

Hess, W. The Anatomy of the Root Canals of the Teeth of the Permanent Dentition, Part 1. New York, William Wood and Co, 1925.

Hsu, Y. Y. \& Kim, S. The resected root surface. The issue of canal isthmuses. Dent. Clin. North Am., 41(3):529-40, 1997.

Kontakiotis, E. G.; Palamidakis, F. D.; Farmakis E. T. \& Tzanetakis, G. N. Comparison of isthmus detection methods in the apical third of mesial roots of maxillary and mandibular first molars: macroscopic observation versus operating microscope. Braz. Dent. J., 21(5):428-31, 2010.

Lima, F. J.; Montagner, F.; Jacinto, R. C.; Ambrosano, G. M. \& Gomes, B. P. An in vitro assessment of type, position and incidence of isthmus in human permanent molars. J. Appl. Oral Sci., 22(4):274-81, 2014.

Pécora, J. D.; Estrela, C.; Bueno, M. R.; Porto, O. C.; Alencar, A. H. G; SousaNeto, M. D. \& Estrela, C. R. A. Detection of root canal isthmuses in molars by map-reading dynamic using CBCT images. Braz. Dent. J., 24(6):569-74, 2013.

Pineda, F. \& Kuttler, Y. Mesiodistal and buccolingual roentgenographic investigation of 7,275 root canals. Oral Surg. Oral Med. Oral Pathol., 33(1):101-10, 1972.

Tam, A. \& Yu, D. C. Location of canal isthmus and accessory canals in the mesiobuccal root of maxillary first permanent molars. J. Can. Dent. Assoc., 68(1):28-33, 2002.

Teixeira, F. B.; Sano, C. L.; Gomes, B. P.; Zaia, A. A.; Ferraz, C. C. \& Souza-Filho, F. J. A preliminary in vitro study of the incidence and position of the root canal isthmus in maxillary and mandibular first molars. Int. Endod. J., 36(4):276-80, 2003.

Versiani, M. A.; Alves, F. R.; Andrade-Junior, C. V.; Marceliano-Alves, M. F.; Provenzano, J. C.; Rôças, I. N.; Sousa-Neto, M. D. \& Siqueira, J. F. Jr. Micro$\mathrm{CT}$ evaluation of the efficacy of hard-tissue removal from the root canal and isthmus area by positive and negative pressure irrigation systems. Int. Endod. J., 49(11):1079-87, 2016.

von Arx, T. Frequency and type of canal isthmuses in first molars detected by endoscopic inspection during periradicular surgery. Int. Endod. J., 38(3):160$8,2005$.

Weller, R. N.; Niemczyk, S. P. \& Kim, S. Incidence and position of the canal isthmus. Part 1. Mesiobuccal root of the maxillary first molar. J. Endod., 21(7):380-3, 1995.

Dirección para Correspondencia:

Ricardo Oliva Rodríguez

Dirección: Av. Manuel Nava 2

Código Postal: 78290

San Luis Potosí, S.L.P.

MÉXICO

E-mail: ricardo.oliva@uaslp.mx

Recibido: 23-01-2017 Aceptado:11-08-2017 